\title{
Laparoscopic treatment of UPJ obstruction in ectopic pelvic kidneys in children
}

\author{
Trattamento laparoscopico dell'Ostruzione del Giunto Pielo-Ureterale nei reni ectopici in età pediatrica
}

\author{
A. Marte,${ }^{1}$ M. Prezioso, ${ }^{1}$ L. Pintozzi, ${ }^{1}$ S. Cavaiuolo, ${ }^{1}$ S. Coppola,${ }^{1}$ M. Borrelli, ${ }^{1}$ P. Parmeggiani ${ }^{1}$
}

Key words: ectopic, kidney, UPJ Obstruction

\begin{abstract}
Aims: To assess the feasibility and safety of a laparoscopic approach to UPJ obstruction (UPJO) in ectopic pelvic kidneys.

Material and Methods: In a retrospective analysis we selected 14 children, aged 6months to 17 years, 12 males, 2 females, who had been treated in our Department between January 2004 and June 2011. 9 patients presented ureteropelvic junction obstruction (in 3 cases pelvic stones coexisted) with normal/moderately reduced $(\geq 25 \%)$ relative function at radionuclide scan (MAG3), 3 nonfunctioning kidneys associated or not to hypertension, 2 congenital hypo-dysplastic kidneys. The evaluation of each patient involved the medical history, ultrasound examination, VCUG, MAG3 diuresis renogram and MRI in some cases. Of the patients presenting UPJO, 5 underwent dismembered pyeloplasty with pyelolithotomy, if required, and 4 pelvic derotation with straightening of the uretero-pelvic junction. A previous cystoscopic placement of a Double J stent was utilized. This facilitated the identification and dissection around the pelvis. With the patient in Trendelenburg position we utilized an umbilical trocar and two trocar in the right and left iliac fossae; an additional trocar, when required, was inserted more cephalad on the midclavear line contralaterally to the lesion. The derotation of ureteropelvic junction was obtained by freeing the kidney's lower pole and by placing intraperitoneally the junction protected with a Double J stent. This was obtained by suturing the peritoneum behind the ureteropelvic junction resulting in a forward rotation of the major axis of the kidney and a straightening of the junction. The 5 patients pre-
\end{abstract}

Pediatric Surgery - Second University of Naples senting nonfunctioning ectopic kidneys underwent laparoscopic nephrectomy. While the removal of congenital hypoplasic kidneys resulted easy, the removal of nonfunctioning kidneys was more difficult due to their complex vascular situation and for the embryonic disposition.

Results: The operating time varied between 40 to 200 minutes. No patient required conversion to open surgery. The hypertension resolved after nephrectomy in all cases. 2 cases of dismembered pyeloplasty required a placement of Double J stent due the recurrence of symptoms and ! patient is waiting for redo operation. The pelvic derotation showed an improvement of diuretic MAG3 renogram and the function remained stable and patiens are symptoms-free.

Conclusion: The UPJO in ectopic pelvic kidneys presents a large spectrum of presentation. The laparoscopic approach provides good surgical exposure, and operative times are acceptable compared to those of laparoscopic procedure in anatomically normal kidneys. It has also proved a very useful tool in the non-functioning kidney nephrectomy thank to the help of magnification in the identification of numerous aberrant vessels that are quite often found in the pelvic kidneys. The derotation of the pelvis seems a useful procedure in moderate obstruction even if a longer followup is needed.

\section{Introduzione}

I reni che non riescono a risalire alla loro posizione normale durante la vita embrionale sono definiti come ectopici. La forma più comune di rene ectopico è il rene pelvico, dove, come suggerisce il nome, il rene rimane nella cavità pelvica. In questi casi l'orientamento del bacinetto è anteriore e la vascolarizzazione si "congela" al momento dell'arresto dell'ascesa presentando una miriade di vasi anomali sia dall'aorta che dalle arterie iliachel.(Fig. 1)

Molti pazienti portatori di ectopia renale rimangono asintomatici tutta la vita ${ }^{2}$. L'incidenza del rene ectopico è approssimativamente di 1 ogni 2200-3000 nati³. Il problema più comune è la ostruzione del Giunto pielo-ureterale (OGPU), quando l'ectopia 


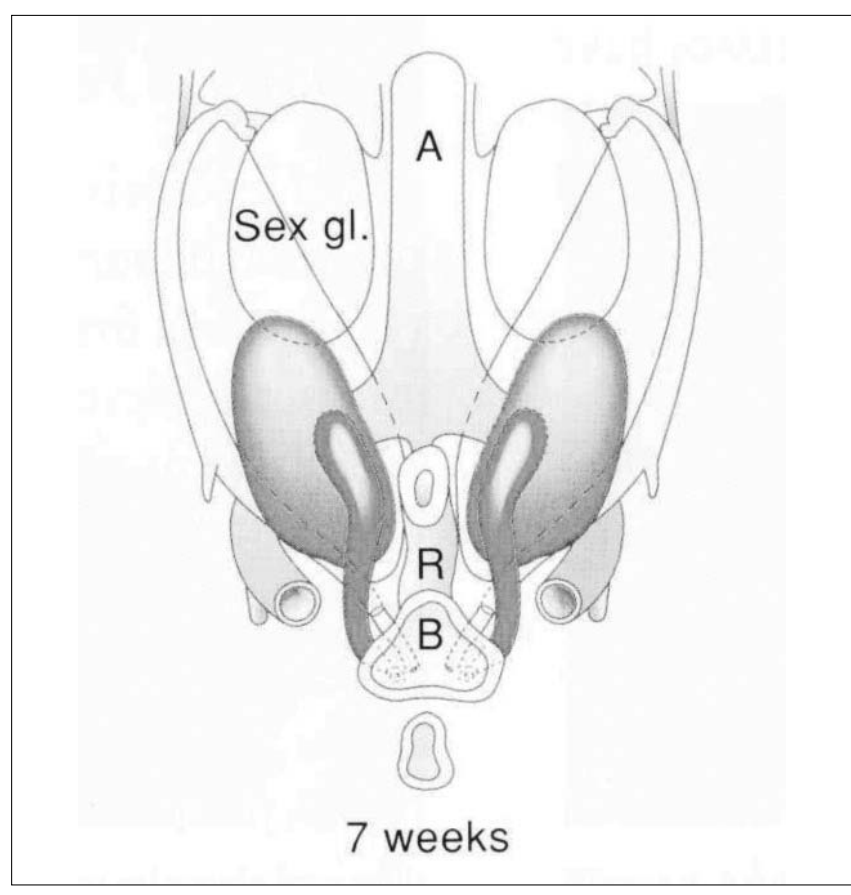

Figure 1.

\section{Materiali e Metodi}

Abbiamo selezionato 14 bambini, di età compresa tra 6 mesi e 17 anni, (12m; 2f) trattati tra il gennaio 2004 e giugno 2011 dallo stesso chirurgo.

Di questi:

- 9 pazienti presentavano ostruzione del GPU (3 casi con calcolosi della pelvi) con normale/moderatamente ridotta funzione relativa alla scintigrafia con MAG3 $(\geq 25 \%)$.

- 3 pazienti presentavano reni ipofunzionanti (funzione relativa $<10 \%), 1$ complicato da ipertensione arteriosa.

- 2 pazienti presentavano ipodisplasia renale congenita.

La valutazione di ogni paziente è stata condotta mediante anamnesi familiare e personale, l'ecografia, Cistografia Minzionale, renogramma diuretico con MAG3 e RMN in casi selezionati.

La sintomatologia prevalente di presentazione è stato dolore addominale peri-ombelicale/basso continuo o intermittente $(10 \mathrm{pz})$, ematuria in 2 casi, IVU 2 pazienti. Nessun paziente presentava RVU alla CUM né ostruzione sottovescicale.

\section{Trattamento}

Tutti i pazienti sono stati operati in posizione supina col fianco sollevato di $20-30^{\circ}$ dal lato affetto. La cistoscopia è sempre stata eseguita prima dell'intervento per la ricognizione dell'ostio ureterale ed il posizionamento di uno stent doppio J per favorire l'identificazione dell'uretere durante la dissezione e favorire il drenaggio renale nel postoperatorio.

E stata eseguita una laparoscopia standard con 3-4 trocars in anestesia generale. Il primo trocar è stato introdotto per via trans-ombelicale "aperta" e due trocars laterali di $3 \mathrm{~mm}$ sono stati introdotti sotto controllo scopico in fossa iliaca destra e sinistra lungo la linea emiclaveare. Se necessario, un quarto trocar è stato introdotto in posi-

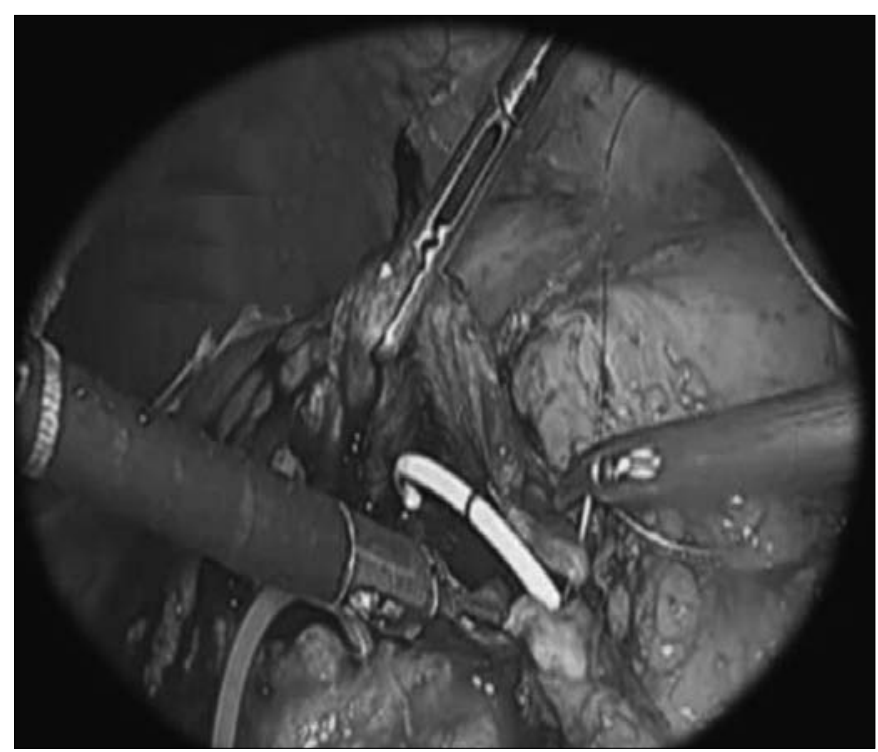




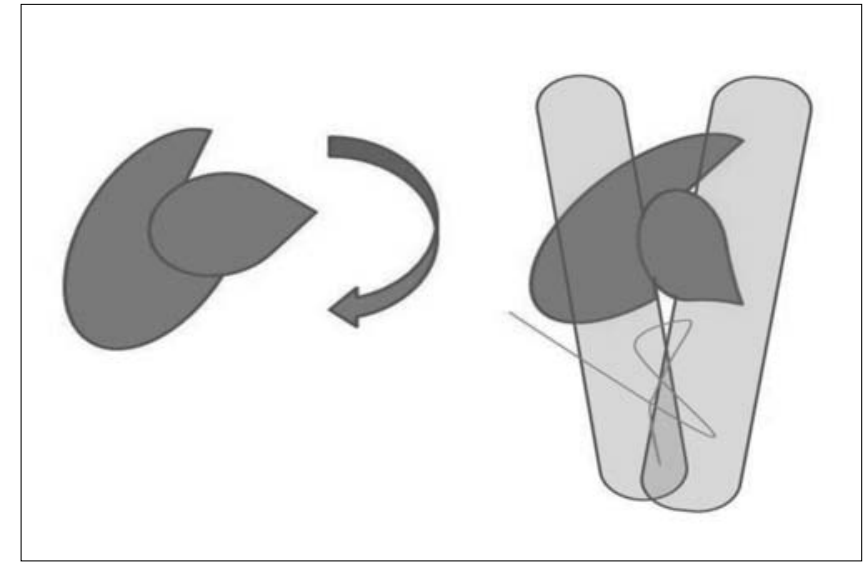

Figure 4.

Schema dell'intervento di derotazione pelvica ed extraperitoneizzazione/raddrizzamento dell'uretere invece di Disposizione embriologica del rene pelvico
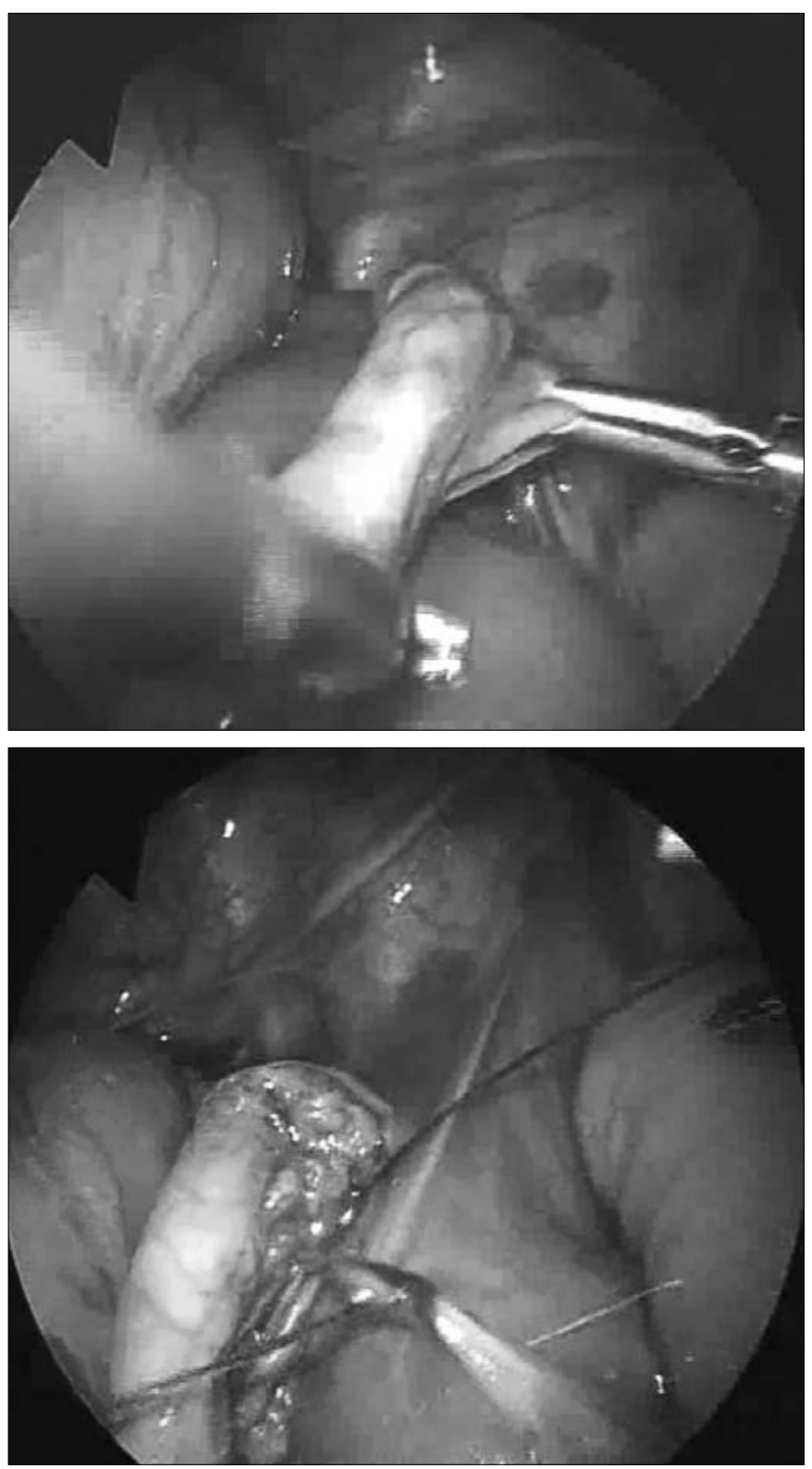

Figure $5,6,7,8$

Tempi laparoscopici della rotazione del rene ectopico e sutura del peritoneo posteriormente all'uretere che assume un decorso rettilineo Pazienti con OGPU : culator . (Fig.2,3)

zione più cefalica a livello della linea medioclaveare destra o sinistra. $\mathrm{Si}$ è così proceduto con un approccio transmesocolico senza mobilitare l'intestino dalla superficie anteriore del rene e della pelvi renale.

- 5 "Dismembered Pyeloplasty" sec. Anderson-Hynes associata o non a pielolitotomia. La pieloplastica è stata eseguita in due emicontinue di maxon 5/0 come pure la riduzione del bacinetto. I calcoli, quando presenti, sono stati estratti sotto visione dopo la pielotomia utilizzando una Johanne da $5 \mathrm{~mm}$ o un Roti-

- 4 derotazioni pelviche con raddrizzamento della giunzione uretero-pelvica. La derotazione è stata ottenuta liberando il polo renale inferiore e suturando il peritoneo dietro la giunzione uretero-pelvica con conseguente rotazione in avanti dell'asse maggiore del rene e un raddrizzamento della giunzione. (Fig. 4-9)
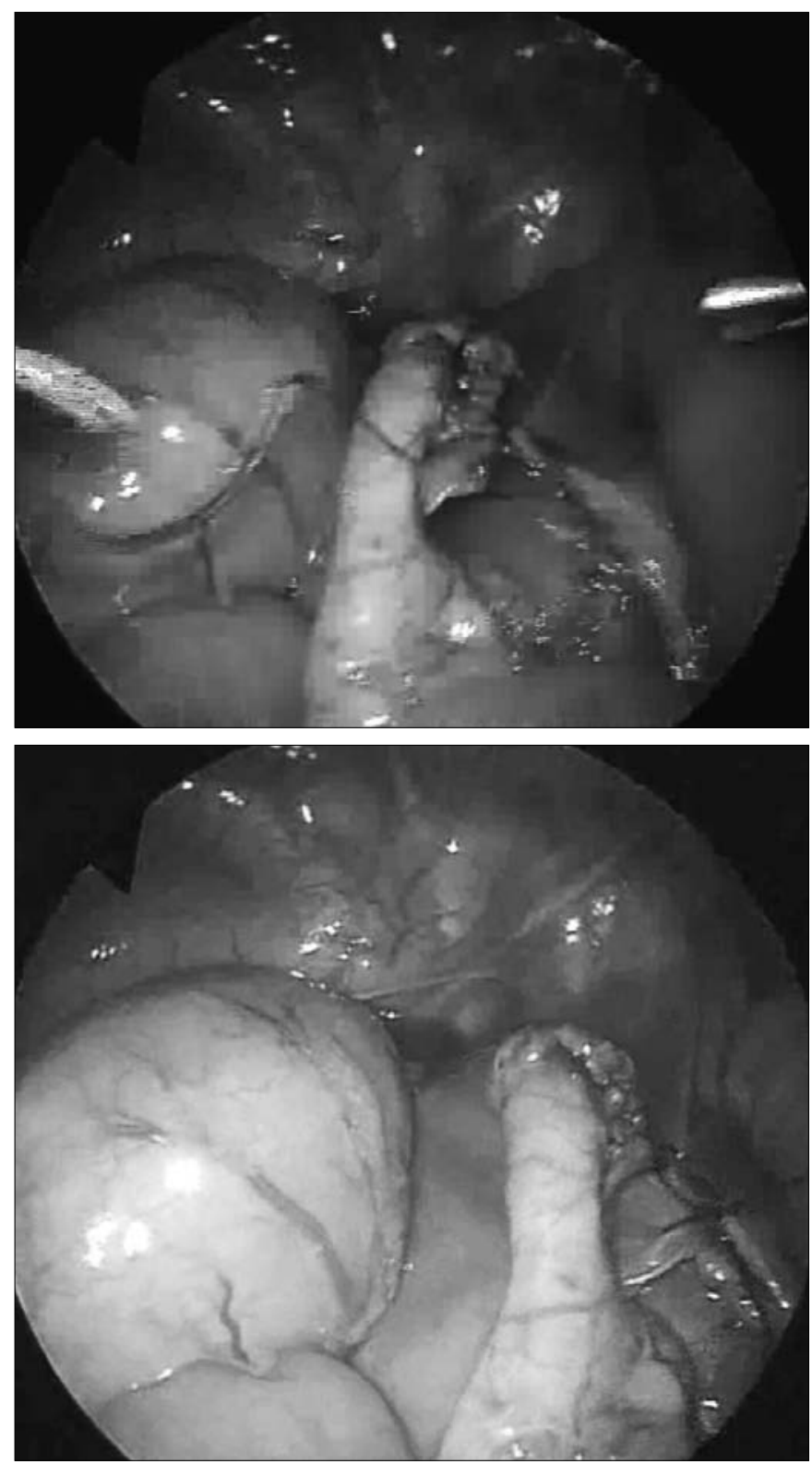
- 5 nefrectomie in pazienti con reni displasici (Fig.10,11) o non funzionanti (Fig. 12,13,14).

Dopo la peritoneizzazione, un drenaggio $12 \mathrm{~F}$ è stato collocato nella cavità peritoneale vicino al rene attraverso uno l'accesso laterale sinistro. Controllata l'emostasi sono stati rimossi i trocars ed eseguito un controllo RX in sala operatoria (soprattutto per i casi di calcolosi).

\section{Risultati}

Il tempo medio operatorio è stato di 170 minuti (range 40200minuti). Il tempo medio di ospedalizzazione è stato di 2.5 giorni (range 1-7). Nessuna complicanza perioperatoria e postoperatoria si è verificata. Le perdite ematiche sono state non rilevanti e nessun paziente ha richiesto trasfusione. In nessun caso è stata necessaria la conversione in chirurgia open. Ad un follow-up medio di 2,8 anni tutti pazienti sono asintomatici.

L'ipertensione si è risolta dopo nefrectomia. Due casi di AndersonHynes hanno richiesto il riposizionamento temporaneo per 6 setti- mane di uno stent doppio J per la ricorrenza dei sintomi. In uno di questi è programmata la revisione chirurgica. I pazienti con calcolosi non hanno presentato recidive. Nei casi in cui è stata effettuata la derotazione della pelvi, si è ottenuto un miglioramento del renogramma alla scintigrafia diuretica con MAG3 e la funzione renale è rimasta stabile con i pazienti asintomatici. Il drenaggio intraperitoneale è stato rimosso dopo 1-2 giorni. I calcoli associati sono risultati costituiti da ossalato di calcio.

\section{Discussione}

L'Ostruzione del GPU può presentarsi come fenomeno isolato o associata ad anomalie renali, e può verificarsi dal $25 \%$ al $33 \%$ dei reni a ferro di cavallo6, e dal $22 \%$ al $37 \%$ di reni ectopici pelvici La chirurgia di questa complessa patologia può essere ablativa 0 ricostruttiva secondo le condizioni del rene.

Nei casi di reni gravemente ipodisplasici la chirurgia ablativa non presenta alcun problema tecnico e la magnificazione laparoscopica è di grande ausilio nell'isolamento e nella asportazione.

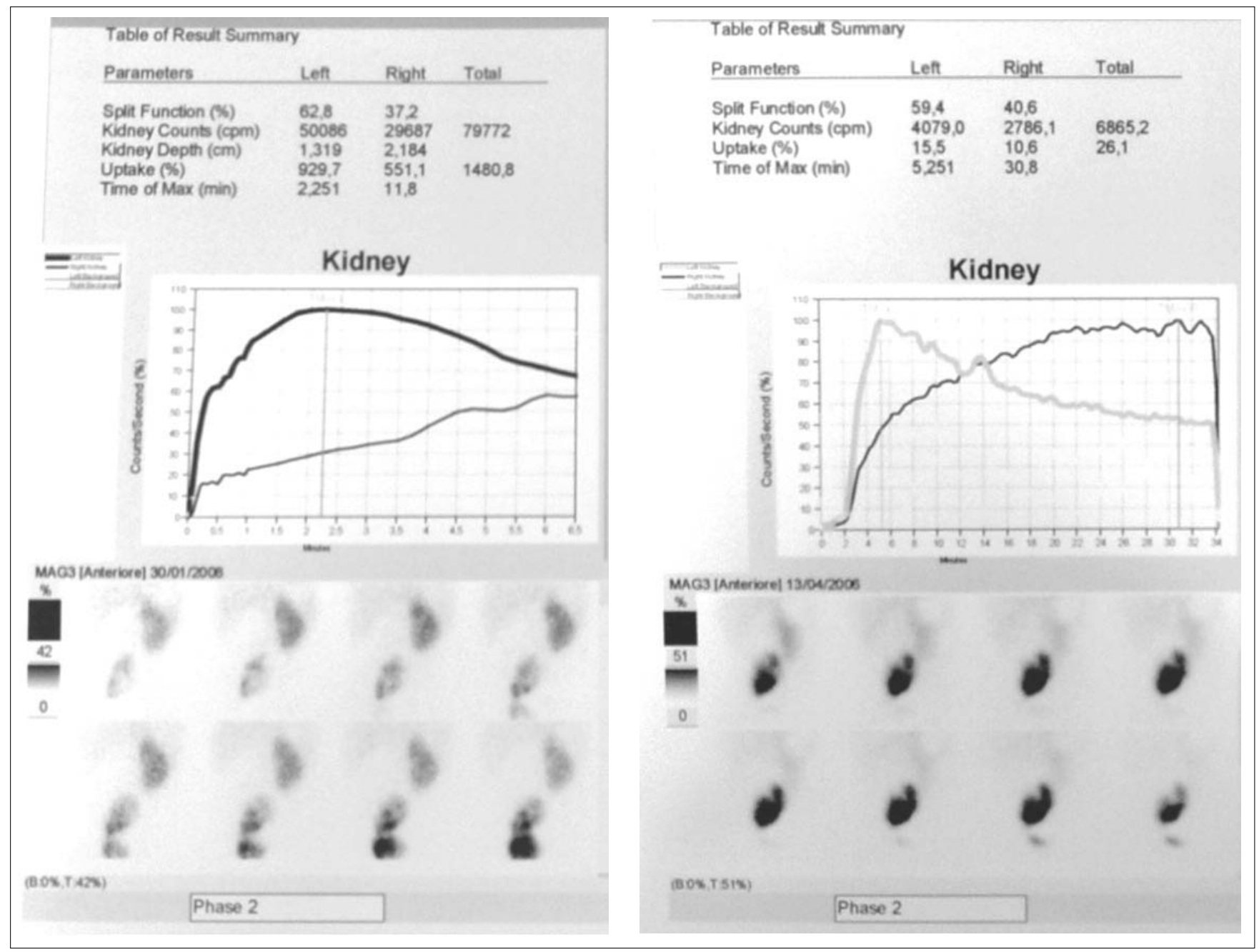



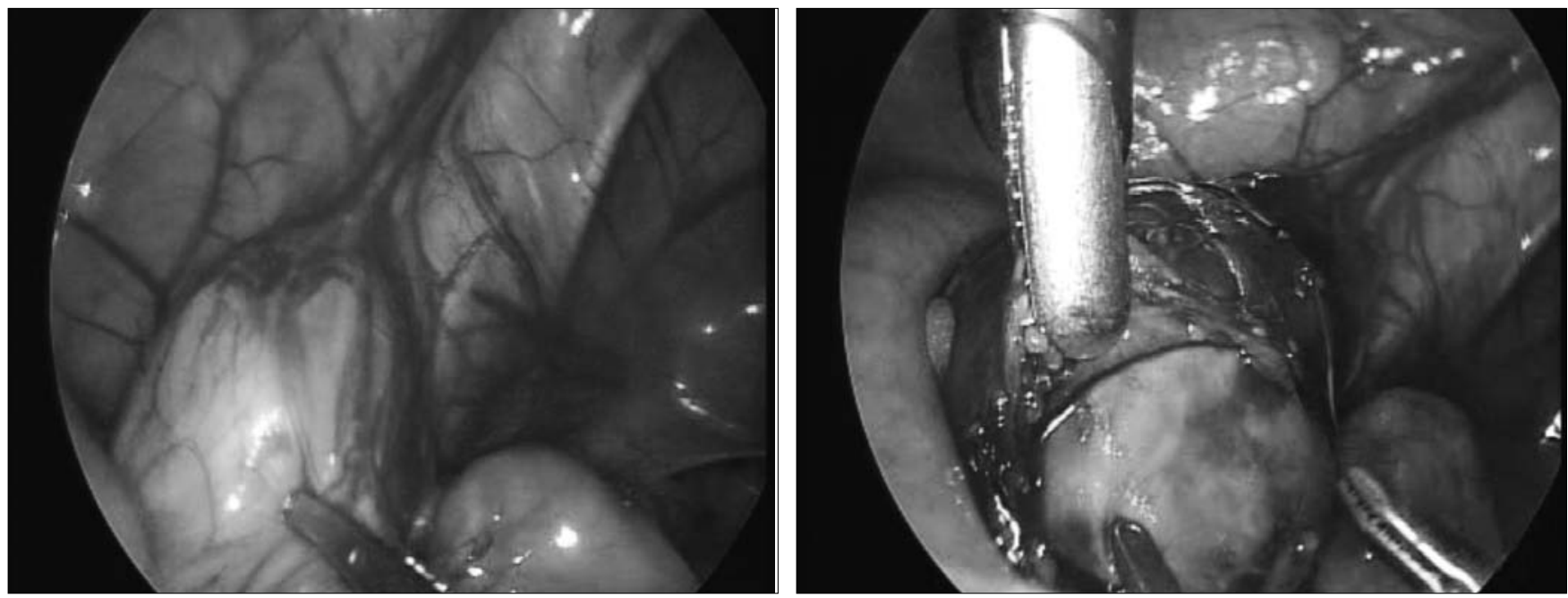

Asportazione di rene ipoplasico congenito ectopico

Figure 10, 11

Più complessa è risultata l'asportazione di reni non funzionanti normoconformati nei quali il problema delle anomalie di vascolari con numerosi reni aberranti può rappresentare un problema di emostasi ${ }^{1}$. La chirurgia ricostruttiva è invece un problema che può richiedere varie soluzioni. Per la posizione del rene pelvico alcuni hanno proposto plastiche differenti dalla Anderson-Hynes quali la flap technique ${ }^{7}$, che presenta una percentuale di successo negli adulti di poco inferiore rispetto alle altre tecniche 8 . In molti casi si trattava di pazienti adulti ed i risultati non sono stati univoci?.

Nel nostro caso abbiamo utilizzato due tecniche: nei casi francamente ostruiti abbiamo utilizzato la pieloplastica sec. Anderson Hynes che ci ha consentito di effettuare anche la nefrolitiotomia. Questa ha avuto successo in 4 casi su 5.

Nei casi invece di idronefrosi intermittente in cui le indagini preoperatorie hanno evidenziato solo un inginocchiamento del GUP abbiamo utilizzato una tecnica conservativa di derotazione del bacinetto che ha consentito di porre il giunto in una posizione pendente e rettilinea in grado di assicurare un buon deflusso di urine.

Quello che risalta dalla nostra casistica è che questa patologia si presenta con uno spettro di sintomi molto ampio che va da una ostruzione intermittente ad una ostruzione franca, spesso associata a calcolosi.

Nei casi inveterati la ostruzione può portare alla perdita della funzione renale, che richiede la nefrectomia, o ad un rene ipoplasico. Su queste condizioni si innesta il problema sintomatologico che nel bambino è spesso sfumato, di difficile interpretazione e solo la consapevolezza della patologia può portare alla diagnosi .

Nei casi più marcati di OGPU il dolore addominale si presenta costante con esacerbazioni nel corso della giornata mentre nei casi intermittenti si associa a periodi di completo benessere. La localizzazione del dolore è risultata prevalentemente periombellicale.

Nei casi, poi, di grave ipodisplasia renale su rene ectopico, i cosiddetti ectopic nephrogenic remnants ${ }^{10}$, la sintomatologia è completamente assente ed il reperto assume il carattere occasionale nel corso di una ecografia addominale. Questi residui dovrebbero essere comunque rimossi per il rischio di trasformazione neoplastica.

Un altro problema collegato alla chirurgia del OGPU del rene ectopico è la complessità dell'intervento.
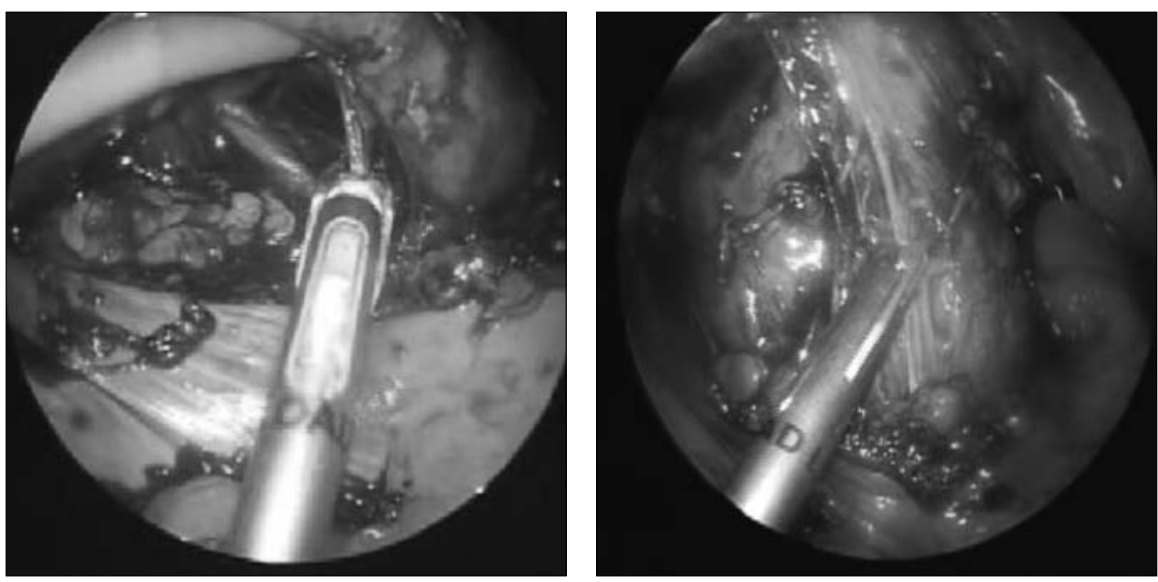

Figure $12,13,14$

Nefrectomia di rene ectopico $\mathrm{dx}$ non funzionante. In evidenza la complessità della vascolarizzazione ed i vasi soprannumerari.

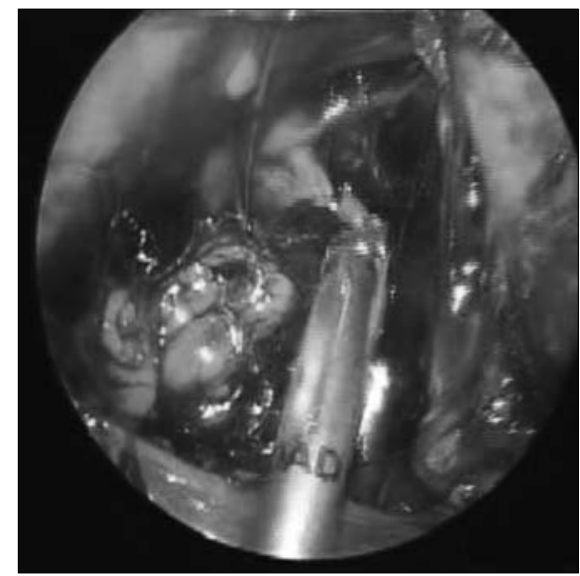


La pieloplastica riduttiva sec Anderson Hynes è stato considerato da sempre il gold standard per il trattamento chirurgico della ostruzione del GPU nei reni altrimenti normali, con un tasso di successo i superiore al $90 \%{ }^{11-12}$. Per i pazienti con un rene a ferro di cavallo il tasso di successo riportato per campi pieloplastica aperta dal $55 \%$ al $80 \%{ }^{13-}$ 14. Nella pieloplastica laparoscopica i tassi di successo sono allo stato attuale pressoché sovrapponibili, ma non vi sono dati sui tassi di successo sulla pieloplastica sec Anderson Hynes nei reni pelvici.

Un altro importante problema è quello della vascolarizzazione ${ }^{15-16}$. Nei reni displasici la rimozione è assolutamente agevole perché la vascolarizzazione si domina in maniera agevole per l'esilità dei vasi: nei nostri casi l'uso del Liga-Sure è stato assolutamente sufficiente ad ottenere una facile emostasi ed asportazione. Differente il caso della nefrectomia su reni precedentemente funzionanti in cui la mancata ascesa del rene comporta la persistenza di una vascolarizzazione assolutamente anarchica con numerosi vasi di origine iliaca che vanno attentamente identificati e clippati pena grosse perdite ematichel.

Un cenno particolare merita, a nostro giudizio l'idronefrosi intermittente da mal posizione pielo-ureterale con sintomi frusti e funzione relativa conservata. In questi ultimi casi, abbiamo constatato che la derotazione del bacinetto e la creazione di un decorso declive della giunzione può essere una soluzione praticabile ed efficace senza ricorrere alla pieloplastica riduttiva; tra l'altro questa derotazione tenderebbe a correggere proprio la malposizione di cui soffre il rene ectopico a causa della sua disposizione embrionale che comporta una disposizione anteriorizzata del bacinetto e del giunto. Questi casi selezionati sulla scorta dei sintomi e sulla base di una dilatazione intermittente del bacinetto tra 10 e $20 \mathrm{~mm}$ hanno risposto bene alla procedura ed i 4 pazienti sono asintomatici e la loro funzione è tuttora stabile. È tuttavia necessario che il follow-up a lungo termine ci possa consentire una più certa definizione dell'efficacia dell'intervento.

\section{Conclusioni}

La chirurgia del rene ectopico pelvico non è sempre semplice ed agevole. La laparoscopia, nella nostra esperienza sembra offrire il vantaggio di una esposizione ottimale grazie alla magnificazione ottica ed una possibilità di fronteggiare tutte le situazioni anomale che l'ectopia renale presenta, in maniera ottimale con il vantaggio della miniinvsività. I tempi operativi e di recupero sembrano peraltro accettabili rispetto a quelli delle procedure laparoscopiche nei reni normoposizionati. Inoltre l'approccio laparoscopico si è rivelato prezioso nel corso della nefrectomia di reni non funzionanti per OGPU per l'identificazione dei numerosi vasi aberranti che sono molto spesso presenti nei reni pelvici. La derotazione del bacinetto da noi attuata sembra una procedura utile ed efficace nei casi di ostruzione intermittente di tipo "mild" anche se un più lungo follow-up è necessario.

\section{Bibliografia}

1 Bauer SB. Anomalies of the upper urinary tract. In: Wein AJ, Kavoussi LR, Novick AC, Partin AW, Peters CA (eds): Campbell Walsh Urology, ed 9. Philadelphia: Saunders Elsevier, 2007, pp 3278-3281.

2 Gleason PE, Kelalis PP, Husmann DA, Kramer SA (1994) Hydronephrosis in renal ectopia: incidence, etiology and significance. J Urol 151(6): 1660-1661

3 Zafar FS, Lingeman JS. Value of laparoscopy in the management of calculi complicating renal malformations. J Endourol 1996;10:379-383.

4 Gleason, P. E., Kelalis, P. P., Husmann, D. A. and Kramer, S. A.: Hydronephrosis in renal ectopia: incidence, etiology and significance. J Urol, 151: 1660, 1994.

5 Pearle MS, Lotan Y. Urinary lithiasis: Etiology, epidemiology, and pathogenesis. In: Wein AJ, Kavoussi LR, Novick AC, Partin AW, Peters CA (eds): Campbell Walsh Urology, ed 9. Philadelphia: Saunders Elsevier, 2007, pp 1389-1391.

6 Segura, J. W., Kelalis, P. P. and Burke, E. C.: Horseshoe kidney in children. J Urol, 108: 333, 1972

7 Basiri A, Mehrabi S, Karami H. Laparoscopic flap pyeloplasty in a child with ectopic pelvic kidney. Urol J. 2010 Jun 10;7(2):125-7

8 Klingler HC, Remzi M, Janetschek G, Kratzik C, Marberger MJ. Comparison of open versus laparoscopic pyeloplasty techniques in treatment of uretero-pelvic junction obstruction. Eur Urol 2003;44:340-5.

9 Bove P, Ong AM, Rha KH, Pinto P, Jarrett TW, Kavoussi LR. Laparoscopic management of ureteropelvic junction obstruction in patients with upper urinary tract anomalies. J Urol 2004;171:77-79.

10 Cooke A, Deshpande AV, La Hei ER, Kellie S, Arbuckle S, Cummins G. Ectopic nephrogenic rests in children: the clinicosurgical implications. J Pediatr Surg. 2009 Dec;44(12):e13-6.

11 Nguyen, D. H., Aliabadi, H., Ercole, C. J. and Gonzalez, R.: Non intubated Anderson-Hynes repair of ureteropelvic junction obstruction in 60 patients. J Urol, 142: 704, 1989

12 Persky, L., Krause, J. R. and Boltuch, R. L.: Initial complications and late results in dismembered pyeloplasty. J Urol, 118: 162, 1977

13 Das, S. and Amar, A. D.: Ureteropelvic junction obstruction with associated renal anomalies. J Urol, 131: 872, 1984

14 Pitts, W. R., Jr. and Muecke, E. C.: Horseshoe kidneys: a 40-year experience. J Urol, 113: 743, 1975

15 Gülsün M, Balkanci F, Cekirge S, Deger A. Pelvic kidney with an unusual blood supply: angiographic findings. Surg Radiol Anat. 22(1):59-61. 2000;

16 Sebe P, Chemla E, Varkarakis J, Latrémouille C. Anatomic variations of the vascularization of the pelvic kidney: apropos of a case and review of the literature. Morphologie. Apr;88(280):24-6. 2004 